\title{
Modelo de reforestación con plantas nativas de la ciénaga de Jaraba mediante la IEP
}

\section{Reforestation model with native plants of the Jaraba swamp through the IEP}

DOI: http://dx.doi.org/10.17981/cultedusoc.9.3.2018.67

Artículo de investigación. Fecha de recepción: 15/06/2018. Fecha de aceptación: 27/11/2018

\author{
Nelcy Camaño-Guerra; Pablo Gutiérrez-Rodero; Javier Ortega-Ramírez; \\ Rosa Gutiérrez-Díaz; Lidiber Pórtela-Villamizar; Fernando Campo-Méndez; \\ Leidis Cáliz-López; Marta Mejía-Yépez; Marlene López-Guerra; \\ Bernardo Acosta-Padilla; Luis Villanueva-Torres; Rafael Bravo-Romero; \\ Luz Vergara-Matute; Eliana López-Pérez y María Núñez-Mercado ${ }^{1}$ \\ IED Antonio Bruges Carmona, sede ERM de Jaraba (Colombia)
}

Para citar este artículo:

Caamaño-Guerra, N., Gutiérrez-Rodero, P., Ortega-Ramírez, J., Gutiérrez-Díaz, R., Pórtela-Villamizar, L., Campo-Méndez, F., Cáliz-López, L., Mejía-Yépez, M., López-Guerra, M., Acosta -Padilla, B., Villanueva-Torres, L., Bravo-Romero, R., Vergara-Matute, L., López-Pérez, E. y Núñez-Mercado, M. (2018). Modelo de reforestación con plantas nativas de la ciénaga de Jaraba mediante la IEP. Cultura. Educación y Sociedad 9(3), 557-566. DOI: http://dx.doi.org/10.17981/cultedusoc.9.3.2018.67

\section{Resumen}

La sequía en época de verano ocasionada por la acción directa del sol sobre las costas a causa de la perdida de vegetación en las mismas, es una realidad que afecta a las poblaciones ribereñas en la actualidad. Los programas de reforestación son herramientas útiles en la mejora de la calidad de vida de las diferentes partes del mundo afectadas por esta realidad. Este estudio tuvo como propósito diseñar un modelo de reforestación con plantas nativas de la ciénaga de Jaraba mediante la Investigación como Estrategia Pedagógica (IEP). La metodología se orientó bajo el enfoque cualitativo, utilizando el tipo de investigación acción, desde un diseño descriptivo, exploratorio. $\mathrm{Pa}$ ra la recolección de datos se utilizaron técnicas como; análisis documental a partir de la implementación de un software que analiza las características de los arbustos y semillas propicias para la reforestación. La población estuvo conformada por cincuenta (50) estudiantes de la IED Antonio Bruges Carmona sede ERM de Jaraba del Municipio de Santa Ana Magdalena. Dando como resultados la creación de un vivero natural con diferentes especies de plantas seleccionadas para este modelo ecológico. Llegando a la conclusión que existe la necesidad de fortalecer la cultura ambiental por parte de los habitantes y visitantes en las orillas de la ciénaga, donde se vierte toda clase de desechos que difícilmente alcanzarán un nivel de degradación.

Palabras clave: modelo de reforestación, plantas nativas, investigación como estrategia pedagógica.

\section{Abstract}

The drought in summer time caused by the direct action of the sun on the coasts because of the loss of vegetation in them, is a reality that affects the riverside populations at present. Reforestation programs are useful tools in improving the quality of life in different parts of the world affected by this reality. The purpose of this study was to design a reforestation model with native plants of the Jaraba swamp through Research as a Pedagogic Strategy (IEP). The methodology was oriented under the qualitative approach, using the type of action research, from a descriptive, exploratory design. For the collection of data, techniques such as; documentary analysis based on the implementation of software that analyzes the characteristics of shrubs and seeds that are conducive to reforestation. The population was conformed by fifty (50) students of the IED Antonio Bruges Carmona ERM headquarters of Jaraba of the Municipality of Santa Ana Magdalena, giving as results the creation of a natural nursery with different species of plants selected for this ecological model. Reaching the conclusion that there is a need to strengthen the environmental culture on the part of the inhabitants and visitors on the banks of the swamp, where all kinds of waste that will hardly reach a level of degradation are poured.

Keywords: reforestation model, native plants, research as a pedagogical strategy. 


\section{Introducción}

Dado que en la actualidad el ambiente se va deteriorando día a día, ya sea por falta de cultura ambiental o por la ampliación de las tierras para la agricultura y pastoreo, cabe decir que, se hace necesario fomentar el cuidado del medio ambiente y la importancia de los árboles para el planeta. Por otro lado, las continuas deforestaciones originan pérdidas del suelo, desaparición de fuentes hídricas e incluso las instalaciones físicas ya sean de vivienda o de otro tipo de las áreas rurales corren el peligro de derrumbarse originando pérdidas humanas.

En efecto, los programas de reforestación rural son herramientas muy útiles en la mejora de la calidad de vida de la población de las diferentes partes del mundo. Cabe destacar que la ONU declaró el 2011 año internacional de los bosques (Organización de las Naciones Unidas para la Alimentación y la Agricultura (FAO, 2011) con el fin de mitigar la deforestación de árboles que se hace evidente tras la tala indiscriminada de los mismos, crear espacios para la ganadería y la agricultura que se practican sin tener en cuenta el desarrollo sostenible. Actualmente los programas de reforestación existentes, no han logrado el éxito esperado debido a que no existe una previa sensibilización y educación ambiental.

A partir de lo realizado por el programa nacional de reforestación de Filipinas, introducido por el gobierno asiático en 2011 se hace la implantación del programa de reforestación, uno de los más extensos y notables del mundo. Filipinas no sólo ha recuperado sus árboles perdidos, sino que los ha multiplicado: 240.000 nuevas hectáreas de bosques, mejorando así el ecosistema. Sin embargo, dicho programa fue duramente criticado por ser implementado con vegetación exótica, por su rápido crecimiento y bajo costo.
Por su parte en Colombia considerado como un país con alto potencial para la reforestación, sin duda alguna, carece de algunos factores y condiciones claves para construir este sector. Actualmente el país posee una masa crítica de bosque plantado, pero ella no logra conformar una parte sólida, como tampoco ha impulsado la forestación ni la creación de una industria forestal relevante que contrarreste este problema.

En el municipio de Santa Ana, corregimiento de Jaraba se encuentra localizada la ciénaga que lleva su mismo nombre, la cual, a causa de factores climáticos, tala, quema indiscriminada de árboles, inundaciones y creación de corrales para la ganadería ha dado como resultado sequías que a su vez ocasionan la pérdida de vida vegetal y animal. Esta ciénaga, mucho tiempo atrás estaba poblada en sus costas por una diversidad de árboles y vegetación como es el caso del mangle "Rhizophora mangle", que es un árbol de hojas grandes y tallo fuerte el cual brindaba su sombra en las orillas de la ciénaga ayudando a mantener sus aguas en tiempo de sequía y preservar gran cantidad de aves y otros animales que moraban en ella. Sin embargo, debido a los comportamientos que afectan el ambiente, poco a poco causaron la perdida de gran cantidad de estas plantas (tabla 1)

Debido a que existen pocos antecedentes sobre trabajos realizados en beneficio a la sociedad, reforestar trae consigo muchos beneficios principalmente el cuidado del ambiente, así como la difusión de la cultura ambiental, de igual manera el beneficio directo de la acción de reforestar, teniendo bases ecológico-sociales en beneficio de la comunidad en general. Reforestar ayudará a la integración en los temas de actualidad, fomentando bases hacia el respeto por el cuidado de la naturaleza, y el trabajo en equipo para su realización. Además de que se pueden vincular tanto 
TABLA 1

Diversidad de vegetación de los mangles de la ciénaga

\begin{tabular}{|c|c|c|c|c|}
\hline $\begin{array}{l}\text { Nombre } \\
\text { común }\end{array}$ & $\begin{array}{l}\text { Nombre } \\
\text { científico }\end{array}$ & Forma & Hábitat & Reforestación \\
\hline Mangle & $\begin{array}{l}\text { Rhizophora } \\
\text { mangle }\end{array}$ & \multirow{3}{*}{$\begin{array}{l}\text { Árbol o arbusto } \\
\text { perennifolio, halófito, } \\
\text { de } 1.5 \text { a } 15 \mathrm{~m} \text { (hasta } \\
30 \mathrm{~m} \text { ) de altura con un } \\
\text { diámetro a la altura del } \\
\text { pecho de hasta } 50 \mathrm{~cm} .\end{array}$} & \multirow{3}{*}{$\begin{array}{l}\text { Especie característica de } \\
\text { los litorales donde forma } \\
\text { a menudo masas puras en } \\
\text { las zonas intermareales de } \\
\text { lagunas costeras y esteros } \\
\text { con influencia de agua } \\
\text { salada. }\end{array}$} & \multirow{3}{*}{$\begin{array}{l}\text { Especie con } \\
\text { potencial para la } \\
\text { reforestación y la } \\
\text { restauración de las } \\
\text { comunidades de } \\
\text { manglar. }\end{array}$} \\
\hline $\begin{array}{l}\text { Mangle } \\
\text { negro }\end{array}$ & $\begin{array}{l}\text { Avicenia } \\
\text { germinans }\end{array}$ & & & \\
\hline $\begin{array}{l}\text { Mangle } \\
\text { blanco }\end{array}$ & $\begin{array}{l}\text { Laguncularia } \\
\text { racemosa }\end{array}$ & & & \\
\hline
\end{tabular}

Fuente: Comisión Nacional para el Conocimiento y Uso de la Biodiversidad, 2017.

los niveles de gobierno municipal y estatal, así como las instancias encargadas del cuidado del ambiente.

Para analizar la influencia del proceso de reforestación, se necesita entender que el crecimiento de un árbol existe en el aumento de sus dimensiones (el diámetro, la altura y el volumen), durante un determinado período de tiempo, siendo la planta una entidad de respuestas multidimensionales, resultado de la interacción entre factores ambientales, por lo que la tarea del silvicultor se encamina al mejoramiento del ambiente de los sitios de plantación Además es pertinente recalcar que los requerimientos básicos de una planta son la disponibilidad de luz, agua, oxígeno, bióxido de carbono y nutrientes, además de otros factores como la interacción biótica y el disturbio y que las plantas perennes crecen en la estación húmeda, mientras que en el verano permanecen sin crecer, por lo que su desarrollo puede medirse en el lapso de un año (Delgado, Murillo y Castillo. 2003).

Cabe decir que, en la República Española, en los años 30 del siglo XX, cuando comenzó a gestarse un plan realista para reforestar el país: "el Plan General para la Repoblación Forestal de España”, que no pudo fraguar hasta concluida la Guerra Civil (1936 - 1939), siendo presentado en 1939 por sus autores Luis Ceballos, Fernández de Córdoba y Joaquín Ximénez de Embún (Gobierno de España, 2015).
Por su parte, la incorporación de árboles, como parte de un programa forestal social, puede tener diferentes funciones, incluyendo las arboledas comunitarias, las plantaciones en el terreno árido, en las vías de pasaje autorizado, alrededor de los terrenos agrícolas, junto a los ríos, lagos, lagunas, ciénagas y humedales, al lado de las casas en las poblaciones cercanas a estas fuentes de agua, todo este tipo de plantación causa impactos ambientales positivos en los mismos y se contribuye a mantener un medio ambiente sano.

Los árboles dan productos útiles y beneficios ambientales y estéticos. Los problemas comunes que surgen de estas actividades son de naturaleza social, puesto que el hombre es el mayor causante de la deforestación en el mundo entero, ya sea directamente cuando los corta para beneficio propio o indirectamente cuando por descuido arroja elementos que pueden causar un desastre forestal o incendio, acabando con miles de hectáreas de bosque y por ende con la fauna que en ellos habita. Desde este punto según la FAO (1999) la Situación de los Bosques del Mundo (SOFO) es que:

En lo que respecta a deforestación, a través de los estudios realizados por el departamento administrativo nacional de estadística - DANE, el Instituto de hidrología, meteorología y estudios ambientales-IDEAM y otras entidades gubernamentales y de orden privado, de hecho, se 
obtiene que, dentro de los porcentajes de deforestación en Colombia, en los últimos dos años ha aumentado considerablemente la tala indiscriminada de árboles, es el caso de la zona del Caribe y de la Amazonia. En el 2010, Colombia ocupaba el tercer puesto a nivel de Suramérica con más territorio boscoso con 59 millones de hectáreas, y el quinto lugar en comparación con otros países con más hectáreas de bosque natural (Fundación para la Educación Superior y el Desarrollo-Fedesarrollo, 2011, p. 3).

Sobre las bases de las consideraciones anteriores, los informes medioambientales recientes se ha demostrado que los países europeos experimentan graves problemas de salud ambiental. Uno de los principales criterios utilizados para dicha investigación es la presencia de árboles en un lapso de más de una década. Suecia mostró una disminución muy significativa en este período de tiempo. Muchos investigadores han utilizado a Portugal como ejemplo para señalar los efectos negativos de la deforestación. Esto se debe a que en los últimos años este país ha experimentado una alta tasa de deforestación; los expertos creen que, si continúa en las próximas décadas, la nación sufrirá los efectos del cambio climático y potencialmente podría perder una gran cantidad de suministro de alimentos y de agua.

Es evidente entonces que, en el modelo de circulación general atmosférica elaborado por el Laboratorio de Ciencias Atmosféricas Goddard se ha demostrado que los grandes cambios en la cubierta vegetal afectan a la lluvia. Por su parte, la vegetación es un factor determinante, sino más bien la correlación entre la humedad del suelo, la vegetación y la energía (fundamentalmente solar) que se necesita para convertir el agua en vapor de agua que forma parte del aire.

De los planteamientos anteriores, los casos en que se ha limpiado la vegetación nativa de crecimiento lento, a un costo económico y social considerable, para reemplazarla con plantaciones de especies exóticas de crecimiento rápido, pero, al final, su productividad fue inferior a la de la vegetación limpiada, lo que quiere decir que, o no se pudo justificar, en relación con los costos. Además, ha habido otros casos en que el comportamiento impredecible de la especie en el ambiente nuevo ha causado el resultado contrario: la sobreproducción. Las especies exóticas pueden convertirse en malezas, difundiéndose en todas partes, desde el sitio de la población, ocupando áreas donde no son deseadas y volviéndose casi imposibles de erradicar.

En efecto, es de suma importancia probar, en forma amplia, la especie exótica (de varias fuentes), antes de utilizarla con profusión en un área nueva. Especialmente, en el caso de las plantaciones de protección, donde sea fundamental establecer y mantener, rápidamente, la cobertura forestal, se debe intercalar entre las especies exóticas de crecimiento rápido, las especies nativas; estas, aunque más lentas, serán más confiables, a la larga. Hechas las consideraciones anteriores, la falta de investigación acerca de las características ambientales y usos finales de muchas de las especies tropicales poco conocidas puede desencadenar desequilibrios climáticos en lo que respecta los cambios en el ambiente.

Cabe decir que, los problemas adicionales en las especies exóticas, posiblemente, no será aceptada, localmente, para el uso final para el cual fue centrada (postes, leña). Una especie que se utiliza, ampliamente, en un lugar para leña, por ejemplo, quizás no será apropiada para otro sitio donde se emplean diferentes alimentos y métodos de cocción. La percepción de la gente, en cuanto a las cualidades de la madera y de los otros productos forestales, puede tener una base cultural y estar firmemente arraigada. Puede ser muy difícil superar los perjuicios, de cualquier índole, contra una variedad. Antes de introducir una especie en un área, se deberá probar su aceptación local (Banco Mundial (1994). 
El precio de la deforestación es altísimo. Los árboles al crecer consumen grandes cantidades de dióxido de carbono, lo que los hace herramientas esenciales para la absorción de las emisiones de gases de efecto invernadero (procedentes de autos, fábricas, centrales de energía y ganado) que dan lugar al cambio climático. Si seguimos perdiendo cubierta forestal, será imposible cumplir el objetivo del Acuerdo Climático de París (Organización de las Naciones Unidas - ONU, 2015) de limitar el calentamiento global a menos de dos grados Celsius (por encima de los niveles preindustriales) antes del 2050. Y lo cierto es que para cumplir el objetivo necesitamos recuperar una cantidad importante de la cubierta ya perdida. Hay dos formas de reforestar. La primera es abandonar a la naturaleza tierras que hoy se usan para la agricultura y esperar que aquella las reconvierta en bosque. Esto no costaría mucho, pero llevaría décadas. La segunda opción es más proactiva: plantar árboles nuevos en abundancia.

Como parte de la Declaración de Nueva York sobre los Bosques (Organización de las Naciones Unidas - ONU, 2014), numerosos gobiernos se comprometieron a restaurar cientos de millones de hectáreas de bosque, pero la mayoría enfrenta restricciones presupuestarias que dificultan financiar las medidas correspondientes, de modo que también se necesitará la participación del sector privado.

Cuando los bosques tienen valor económico, es más probable que sean cultivados antes que destruidos. De hecho, hace milenios que la humanidad cultiva árboles por lucro; hoy, los bosques productivos cubren un área de más de 1.000 millones de hectáreas, es decir alrededor de la cuarta parte de la superficie boscosa del mundo.

Los bosques ya han desaparecido en muchas partes del mundo, y los índices de deforestación mundial llegan hasta 15 millones de hectáreas por año. En la mayor parte del mundo la deforestación se aceleró durante la década de los noventa. En este sentido conviene destacar que los índices de deforestación tienden a oscurecerse por la ambigüedad que existe en torno a la definición del bosque.

Cabe agregar que, en la actualidad, estos trabajos de preparación incluyen el subsolado del suelo, pero la preparación es puntual para cada pie a implantar. La cobertura arbórea también ayuda a reducir el flujo rápido de las aguas regulando de esta manera el caudal de las fuentes hídricas, mejorando la calidad del agua y reduciendo la entrada de sedimentos a las mismas, mantiene la humedad en épocas de verano y se preserva el ecosistema.

Con la repoblación de la ciénaga de Jaraba con especies nativas se busca contribuir a que la comunidad y a la fuente hídrica brinden un gran beneficio, puesto que con la presencia de árboles en sus orillas se podrá disfrutar de un aire más puro y un ambiente más sano, así como el mantener la fuente acuífera con buenos niveles pese a que se esté padeciendo de verano o época de sequía, es decir que, con frecuencia, el lugar donde son sembrados los árboles, plantados o silvestres, pertenecen, oficialmente, al estado, lo cual desalienta la reforestación. Además, al sembrar en un terreno que se suponía era comunitario, cuando, en realidad, ciertas personas tienen derechos sobre el mismo, los productos destinados al beneficio de los miembros de la comunidad, serán reclamados por un número reducido de gente, a menudo, la menos necesitada.

En efecto, se requiere una transformación radical de comportamiento para poder cambiar la recolección tradicional de la leña y otros productos naturales, con el cultivo de los mismos bosques. El personal forestal, generalmente, ha sido capacitado para manejar bosques naturales, y tienen la responsabilidad de proteger los bosques comunales; pero, a menudo, no consiguen 
la confianza de la gente rural, ni poseen las destrezas de comunicación y análisis de sistemas sociales, que requieren para el trabajo de forestación social. Como resultado, surgen muchos problemas.

Por otro lado, es frecuentemente, se siembran ciertas especies porque las semillas están disponibles, y no por su uso final o la idoneidad del sitio. Una vez establecidas las plantaciones, la gente no sabe cómo cuidarlas, cuando ralearlas o cosecharlas, ni qué hacer con los árboles, una vez cortados. Las plantaciones que se sembraron para un propósito, pueden, eventualmente, ser utilizadas para otro, o no encontrarán uso, porque no existe ningún plan claro. A menudo, la gente que durante generaciones ha cortado los árboles para aumentar su terreno agrícola, no entiende los beneficios de los proyectos forestales que disminuyen tierra de la agricultura. Las actividades locales de reforestación, tales como las arboledas comunitarias y la siembra de árboles alrededor de las residencias, pueden tener muchos beneficios directos para los individuos y las comunidades.

Cabe agregar que, las especies nativas, a menudo, crecen más lentamente que las exóticas, pero, ordinariamente, son más viables a largo plazo; han sido seleccionadas y refinadas, genéticamente, durante siglos, y se han adaptado a las condiciones locales, por eso, están mejor preparadas para sobrevivir los extremos climáticos y brotes de plagas y enfermedad locales. Los impactos negativos de la preparación del sitio incluyen, no sólo la pérdida de la vegetación existente y los valores ambientales, económicos y sociales que ésta pueda tener, sino también los problemas ambientales relacionados con el desbroce de la tierra: la mayor erosión, la interrupción del ciclo hidrológico, la compactación del suelo, la pérdida de nutrientes y la disminución consiguiente en la fertilidad del suelo. De acuerdo con Canivel, J.(2014), los árboles nativos son mejores por estas razones: a. Están mejor adaptados a las condiciones climáticas locales ya que, naturalmente, crecen en el país en primer lugar. Esto los hace más fuertes y más resistentes a largo plazo.

b. Tienen una mayor resistencia a las plagas y los tifones. En Albay, los árboles exóticos cayeron después del paso del tifón Yolanda mientras los árboles nativos, aunque perdieron muchas de sus hojas permanecieron en pie.

c. Son más efectivos para promover la biodiversidad. Algunos tipos de pájaros no hacen nidos en árboles exóticos. En algunos casos, el sotobosque no crece donde se plantan árboles exóticos. Los animales nativos también son más propensos a comer los frutos de los árboles autóctonos.

d. Muchos árboles nativos de Filipinas son árboles de alto valor que pueden tener buenos precios de mercado, y finalmente contribuyen al componente de alivio de la pobreza del PNG, que busca proporcionar medios de subsistencia para las comunidades a través de los productos forestales.

e. Los árboles nativos son los tipos de árboles plantados por la mayoría de los pueblos indígenas y grupos comunitarios. Si el New Generation Plantations - NGP prioriza los árboles nativos, estos grupos obtendrán los beneficios. En comparación, los árboles exóticos generalmente provienen de proveedores comerciales (Canivel, 2014)

\section{Metodología}

Se realizó un estudio de tipo cualitativo, utilizando el modelo de investigación acción, desde un diseño descriptivo, exploratorio. La investigación cualitativa busca conocer e interpretar la realidad de los participantes a través de sus propias experiencias, entregando una información 
subjetiva del fenómeno de estudio (Hernández, Fernández, y Baptista, 2010). Por su parte el modelo de investigación acción plantea una producción de conocimiento basado en la reflexión de los participantes, teniendo en cuenta la participación activa de maestros en el proceso de enseñanza y acompañamiento tecnológico, guiando al estudiante en su proceso de investigación. Generando nuevo saber y conocimiento sobre una realidad determinada en un proceso de aprendizaje colaborativo.

Los estudios con un diseño descriptivo están encaminados a especificar las características de la población sujeto de estudio o los fenómenos que sean sometidos a análisis. Bajo un estudio de tipo exploratorio, debido a que no se han realizado estudios previos en la institución. (Hernández, Fernández, y Baptista, 2010). La población estuvo conformada por cincuenta (50) estudiantes de la IED Antonio Bruges Carmona sede ERM de Jaraba del Municipio de Santa Ana Magdalena

El instrumento de recolección de información empleado fue un software educativo interactivo basado en el sistema operativo Windows identificado como "ambientalistas" en donde los estudiantes identifican las principales causas de la problemática ambiental ayudando a reconocer las plantas nativas de la zona costera colombiana y cuáles son las mejores opciones con las cuales se puede llegar a reforestar esta zona costera, específicamente la Ciénaga de Jaraba en el municipio de Santa Ana Magdalena.

\section{Resultados}

A partir de la utilización del software "ambientalistas" se diseñaron viveros con flora seleccionada por sus características de resistencia a la humedad y al agua, tales como el uvero, el totumo, el anón cienaguero, la uva de lata, el suán, Palo prieto (ver tabla 2) entre otras, destinadas a la utilización del proceso de recuperación del ecosistema de las costas de la ciénaga de Jaraba del municipio de Santa Ana Magdalena; ya que la siembra de árboles estabiliza el suelo, reduce la erosión de las laderas, purifica el aire, mejora la calidad del agua de la fuente hídrica, cuida la fauna, brinda sustento a la comunidad en cuanto de sus ramas pueden ser utilizadas como recurso de combustible para cocer sus alimentos ya que no poseen gas natural siempre y cuando lo hagan moderadamente.

TABLA 2

Vegetación y reforestación de la ciénaga de Jaraba

\begin{tabular}{|c|c|c|c|c|}
\hline $\begin{array}{l}\text { Nombre } \\
\text { común }\end{array}$ & $\begin{array}{l}\text { Nombre } \\
\text { científico }\end{array}$ & Forma & Hábitat & Reforestación \\
\hline Uvero & $\begin{array}{l}\text { Coccoloba } \\
\text { uvifera }\end{array}$ & $\begin{array}{l}\text { Este es un Árbol o arbusto } \\
\text { perennifolio, de } 9 \text { a } 15 \mathrm{~m} \text { de } \\
\text { altura con un diámetro a la } \\
\text { altura del pecho de hasta } 1 \mathrm{~m} \text {. } \\
\text { La planta varía grandemente } \\
\text { en tamaño: desde arbustos bajos } \\
\text { postrados en playas azotadas } \\
\text { por el viento a árboles pequeños } \\
\text { de ramas apartadas. Copa / } \\
\text { Hojas. Copa redondeada. Hojas } \\
\text { alternas, }\end{array}$ & $\begin{array}{l}\text { Esta especie se encuentra } \\
\text { limitada a las playas } \\
\text { arenosas y rocosas y a } \\
\text { las espesuras de la costa. } \\
\text { Requiere de un clima } \\
\text { tropical o subtropical. }\end{array}$ & $\begin{array}{l}\text { Especie con } \\
\text { potencial } \\
\text { para } \\
\text { reforestación } \\
\text { productiva } \\
\text { en zonas } \\
\text { degradadas } \\
\text { de selva. }\end{array}$ \\
\hline
\end{tabular}




\begin{tabular}{|c|c|c|c|c|}
\hline $\begin{array}{l}\text { Nombre } \\
\text { común }\end{array}$ & $\begin{array}{l}\text { Nombre } \\
\text { científico }\end{array}$ & Forma & Hábitat & Reforestación \\
\hline Totumo & $\begin{array}{l}\text { Crescentia } \\
\text { cujete }\end{array}$ & $\begin{array}{l}\text { Arbol o arbusto caducifolio de } \\
4 \text { a } 8 \mathrm{~m} \text { (hasta } 18 \mathrm{~m} \text { ) de altura } \\
\text { con un diámetro a la altura del } \\
\text { pecho de hasta } 30 \mathrm{~cm} \text {; algunos } \\
\text { ejemplares llegan a los } 60 \mathrm{~cm} .\end{array}$ & $\begin{array}{l}\text { Prospera en áreas abiertas } \\
\text { tipo sabana, propio de } \\
\text { tierras planas bajas, } \\
\text { cañadas, en las selvas } \\
\text { bajas subcaducifolias que } \\
\text { bordean los lechos secos } \\
\text { de arroyos y en las selvas } \\
\text { bajas caducifolias que } \\
\text { cubre las serranías. }\end{array}$ & $\begin{array}{l}\text { Especie con } \\
\text { potencial } \\
\text { para } \\
\text { reforestación }\end{array}$ \\
\hline $\begin{array}{l}\text { Anón } \\
\text { cienaguero }\end{array}$ & $\begin{array}{l}\text { Annona } \\
\text { squamosa }\end{array}$ & $\begin{array}{l}\text { Arbol o arbusto perennifolio / } \\
\text { caducifolio, de } 3 \text { a } 8 \mathrm{~m} \text { (hasta } 10 \\
\text { m) de altura. }\end{array}$ & $\begin{array}{l}\text { Abundante a lo largo de } \\
\text { pendientes fragosas de } \\
\text { barrancas y cañadas. } \\
\text { Prospera en acahuales, } \\
\text { relictos de selva media, } \\
\text { claros de floresta, cafetales } \\
\text { y a lo largo de caminos. } \\
\text { Crece en suelos derivados } \\
\text { de material calizo o ígneo } \\
\text { y metamórfico, pero } \\
\text { siempre en suelos con buen } \\
\text { drenaje y buena capacidad } \\
\text { de retención de humedad. }\end{array}$ & $\begin{array}{l}\text { Especie con } \\
\text { potencial } \\
\text { para } \\
\text { reforestación } \\
\text { productiva } \\
\text { en zonas } \\
\text { degradadas } \\
\text { de selva. }\end{array}$ \\
\hline $\begin{array}{l}\text { Uva de } \\
\text { lata }\end{array}$ & $\begin{array}{l}\text { Bactris } \\
\text { guineensis }\end{array}$ & $\begin{array}{l}\text { Tallo cespitoso } 5 \text { m de alto y } 4 \\
\text { cm DAP. Pinnas subopuestas, } \\
\text { linear-lanceoladas. Raquillas } \\
\text { 8-30. Fruto purpúreo-negruzco }\end{array}$ & $\begin{array}{l}\text { Se encuentra en zonas } \\
\text { de bosque seco desde } \\
\text { Nicaragua, a lo largo } \\
\text { de la Costa Pacífica de } \\
\text { Centroamérica, hasta el } \\
\text { norte de Venezuela. En } \\
\text { Colombia se encuentra } \\
\text { ampliamente distribuida } \\
\text { en toda la región Caribe. }\end{array}$ & $\begin{array}{l}\text { Especie con } \\
\text { potencial } \\
\text { para } \\
\text { reforestación }\end{array}$ \\
\hline Suán & $\begin{array}{l}\text { Ficus } \\
\text { dendrocida }\end{array}$ & $\begin{array}{l}\text { Árbol semideciduo con una } \\
\text { corona baja, densa y ancha; } \\
\text { puede crecer de } 8 \text { a } 30 \text { metros } \\
\text { de altura. El tronco puede tener } \\
\text { entre } 40 \text { y } 60 \mathrm{~cm} \text { de diámetro. } \\
\text { La planta a menudo produce } \\
\text { muchas raíces aéreas. }\end{array}$ & $\begin{array}{l}\text { Selva amazónica y bosque } \\
\text { de sabana, en suelos } \\
\text { sujetos a inundaciones } \\
\text { estacionales, favoreciendo } \\
\text { las condiciones húmedas } \\
\text { y fértiles. Márgenes de } \\
\text { pantanos de mareas en } \\
\text { Panamá. }\end{array}$ & $\begin{array}{l}\text { Especie con } \\
\text { potencial } \\
\text { para } \\
\text { reforestación }\end{array}$ \\
\hline $\begin{array}{l}\text { Palo } \\
\text { Prieto }\end{array}$ & $\begin{array}{l}\text { Vauquelinia } \\
\text { corymbosa }\end{array}$ & $\begin{array}{l}\text { Arbolito o arbusto de copa } \\
\text { redondeada; ramas jóvenes } \\
\text { tomentulosas; peciolo de (1.4)2.5 } \\
\text { a } 5.5(8) \mathrm{cm} \text { de largo, lámina } \\
\text { foliar oblongo-lanceolada o } \\
\text { angostamente lanceolada a } \\
\text { elíptica, oblonga o anchamente } \\
\text { ovada, de (4)6 a } 10(11.5) \mathrm{cm} \\
\text { de largo, margen agudamente } \\
\text { serrado, a veces doblemente } \\
\text { serrado, con } 6 \text { a } 10(14) \text { dientes } \\
\text { por } 5 \mathrm{~cm} \text {, de textura coriácea. }\end{array}$ & $\begin{array}{l}\text { Especie polimorfa, } \\
\text { habitante de ambientes } \\
\text { semiáridos y áridos. }\end{array}$ & $\begin{array}{l}\text { Especie con } \\
\text { potencial } \\
\text { para } \\
\text { reforestación }\end{array}$ \\
\hline
\end{tabular}

Fuente: Comisión Nacional para el Conocimiento y Uso de la Biodiversidad, 2017. 
Estas actividades de reforestación impulsan la acción ciudadana en defensa del medio ambiente, participando en acciones forestales, sensibilizando a la población, incentivando la participación social y promoviendo la educación ambiental de las zonas costeras. En efecto, las reforestaciones participativas son plantaciones organizadas por asociaciones de voluntariado ambiental, centros educativos, ayuntamientos, etc. con el objetivo de mejorar, restaurar y conservar espacios naturales degradados.

Adicional al software ambientalistas y a los viveros con plantas también se realizó una observación registrada y detallada en las costas de la Ciénaga de Jaraba con los siguientes resultados:

a. En la ribera del margen izquierdo de la ciénaga se observan distintos factores contaminantes que afectan el funcionamiento y la vida misma, como son los desechos urbanos y residuos que no son biodegradables. Cabe decir que, este representa un gran peligro para la poca vida de los peces que están en la ciénaga; además de esto, las aguas negras que se producen en Santa Ana, en su gran mayoría las de los establecimientos comerciales y particulares, se vierten todo hacía la ciénaga, haciendo que la contaminación aumente progresivamente. De hecho, se observa el vertimiento de escombros y muchos materiales plásticos como los cauchos y las llantas por gran parte de la orilla de la ciénaga, un lugar no estipulado por la alcaldía para la disposición de escombros; y se evidencian muchos envases de botellas y bolsas llenas con basuras.

- La parte derecha de la ciénaga de Jaraba tiene puestos de venta de toda clase de pescado de la población y no siguen ninguna norma higiénica con respecto al cuidado hacia la ciénaga; arrojando desechos orgánicos a la misma y así produciendo más contaminación ambiental en esta zona.
- Al no existir un ente gubernamental encargado de vigilar y controlar los recursos naturales, las personas y habitantes de las riberas del río no toman conciencia del problema que causa tirar las basuras y no saben el daño incalculable que representa este mal hábito.

\section{Conclusiones}

A partir de las visitas de la zona se puede concluir que la ciénaga de Jaraba no ha sido ajena a la tala indiscriminada de los bosques que rodean las costas del Atlántico, adicionado a los fenómenos climáticos que han contribuido al deterioro de la misma, identificándola como uno de los orígenes de la deforestación que se ha presentado en los alrededores de la ciénaga, contribuyendo con el deterioro de las especies nativas y no nativas de este medio. Estos han sido la principal causa de esta emergencia ecológica incluyendo a la deforestación y los desechos urbanos por la acción humana sobre la naturaleza que han destruido la superficie forestal, originando un inmenso daño a la calidad de los suelos.

Los estudios de campo y las asesorías de agrónomos, han determinado que las plantas escogidas por el grupo de investigación para la actividad de reforestación son apropiadas no solo por ser nativas sino porque son especies que resisten grandes periodos dentro del agua caudada por inundaciones y a la humedad las cuales pueden contribuir a solucionar este problema ambiental que viene padeciendo esta ciénaga, mejorar nuestro entorno, recurso fluvial, medio ambiente y la población en general.

La implementación de la Investigación como estrategia pedagógica para la recopilación de la información ha sido idónea a la necesidad de un modelo de reforestación adecuado para el problema ambiental que padece esta fuente hidrográfica tan importante para el departamento de Magdalena, como es la Ciénaga de Jaraba. 


\section{Referencias}

Banco Mundial (1994). Libro de Consulta para Evaluaci6n Ambiental. I, II y III. Recuperado de http://documentos.banco$\mathrm{m} \mathrm{und} \mathrm{i} \mathrm{a} \mathrm{l.org} \mathrm{/} \mathrm{c} \mathrm{u} \mathrm{r} \mathrm{a} \mathrm{t} \mathrm{e} \mathrm{d} \mathrm{/}$ es / $472401468161955207 / \mathrm{pdf} /$ WTP1390SPANISH10Box338902B01PUBLIC1.pdf

Cabello-Eras, J. (2016). Acercamiento a la producción más limpia como estrategia de gestión ambiental. International Journal of Management Sciences and Operations Research, 1(1), 4-7. Recuperado de http://ijmsoridi.com/index.php/ijmsor/article/view/71

Canivel, J. (2014). Is the gov't reforestation program planting the right trees? Recuperado de https:/www.rappler.com/ nation/51200-national-greening-program-native-trees

Comisión Nacional para el Conocimiento y Uso de la Biodiversidad. (2017). Species Plantarum. 1(443). 1753. Recuperado de http://www.conabio.gob.mx/conocimiento/info_especies/arboles/doctos/58-rhizo1m.pdf

Delgado, A. Murillo, M. y Castillo, M. (2003). Crecimiento de especies forestales nativas en la zona norte de Costa Rica. Agronomía Costarricense. 27(1). 63-78.

FAO. (2011). Año Internacional de los Bosques. Recuperado de http://www.fao.org/ docrep/013/i2015s/i2015s.pdf

FAO. (1999). Situación de los Bosques del Mundo. Recuperado de http://www.fao. org/docrep/W9950S/W9950S00.htm

Fedesarrollo. (2011). Deforestación en Colombia: Retos y perspectivas. Recuperado de http://www.repository.fedesarrollo. org.co/bitstream/handle/11445/337/ KAS\%20SOPLA_Deforestacion\%20 en $\% 20$ Colombia $\% 20$ retos $\% 20 y \% 20$ perspectivas.pdf? sequence $=2 \&$ isAllowed $=y$
Gobierno de España. Ministerio de Agricultura, alimentación y medio ambiente. (diciembre 8,2015 ). $75^{\circ}$ aniversario Plan general de reforestación de España. [Digital Extremadura]. Recuperado de https://digitalextremadura.com/ P61360/

Hernández, R., Fernández, C. y Baptista, P. (2010). Metodología de la investigación. México, D.F.: Mc Graw Hill.

ONU. (2014). Avances de la Declaración de Nueva York sobre los Bosques. Recuperado de http://forestdeclaration.org/wp$\begin{array}{lllllll}\text { c } & 0 & n & t & \text { e } & n & t\end{array}$ uploads/2015/10/Avances_de_la_ Declaración_de_Nueva_York_sobre_ los_Bosques-Resumen_Ejecutivo30-11-15-ES.pdf

ONU. (2015). Acuerdo de París. Recuperado de https://unfccc.int/files/meetings/ paris_nov_2015/application/pdf/paris_ agreement_spanish_.pdf

República de Colombia. Minambiente. (2016). 100 plantas del Caribe Colombiano. Bogotá, D.C.: Fondo Patrimonio Natural. Recuperado de http://pdf. usaid.gov/pdf_docs/PA00MDS4.pdf

Segura, E. (2016). Información, estabilidad y complejidad de aprendizaje en memorias asociativas. International Journal of Management Sciences and Operations Research, 1(1), 49-53. Recuperado de http://ijmsoridi.com/index.php/ijmsor/article/view/77

Sepúlveda, A., Martínez, R., Medina, S. y Salazar, F. (2016). Propuesta de diseño de una red supply chain para la agrocadena de cacao, municipio de Viotá, Cundinamarca. International Journal of Management Sciences and Operations Research, 1(1), 35-42. Recuperado a partir de http://ijmsoridi.com/index. php/ijmsor/article/view/75 